\title{
Geometrical multifractality of the perimeter of DLA clusters
}

\author{
William G. Hanan $^{\mathrm{a}, *}$, Daniel M. Heffernan ${ }^{\mathrm{a}, \mathrm{b}}$ \\ a Department of Mathematical Physics, National University of Ireland Maynooth, Maynooth, Co.Kildare, Ireland \\ b School of Theoretical Physics, Dublin Institute for Advanced Studies, Dublin 4, Ireland
}

\begin{abstract}
The geometrical multifractality of diffusion-limited aggregation (DLA) clusters is investigated by evaluating the $D_{q}$ spectrum for $q \geqslant 0$ using the standard box-counting technique. Using the cluster points themselves as input to the algorithm, deviations were found from the expected multifractal scaling. However on examining the geometric scaling properties of the cluster perimeter, such deviations were found to be significantly reduced, thus allowing a reliable $D_{q}$ spectrum to be calculated. (C) 2000 Elsevier Science Ltd. All rights reserved.
\end{abstract}

In an early work on the geometrical multifractality of diffusion-limited aggregation (DLA) clusters, $D_{q}$ spectra were obtained for clusters grown in a strip geometry yielding values of $D_{q}=1.60 \pm 0.02$ and $D_{q}=1.57 \pm 0.05$ for $q \geqslant 0[1,2]$. This was a point of some controversy as these results were inconsistent with the well established value of $1.71 \pm 0.02$ for the dynamical dimension $D_{\mathrm{d}}$ obtained from the dependence of the radius of gyration $R_{\mathrm{g}}$ on $M$, the cluster mass [3]. Gang Li et al. argued that the $D_{q}$ spectra suffered from finite-size effects and that with an appropriate correction to scaling, the result $D_{q}=1.69 \pm 0.03$ was obtained, consistent with the value of $D_{\mathrm{d}}[2]$. In this paper, using the standard boxcounting technique we examine the geometrical multifractality of DLA clusters (grown in a circular geometry) and their perimeters.

The generalised dimensions $D_{q}$ for $q \geqslant 0$ were estimated using the standard box-counting algorithm. This involves taking a cluster of linear extension $L$, covering it with a grid of boxes of size $l$ and calculating the partition function $Z_{q}=\sum P_{i}(\epsilon)^{q}$, where the summation is taken over all the boxes covering the cluster, $\epsilon$ is the dimensionless parameter defined as the ratio $l / L$ and $P_{i}(\epsilon)$ is the fraction of data points in box $i$. The values of $D_{q}$ are obtained from the scaling of $Z_{q}$ with $\epsilon$. It is expected that $Z_{q} \sim \epsilon^{(q-1) D_{q}}$ over some intermediate range of $\epsilon$. As the location of the grid is arbitrary, the quantity $\ln Z_{q}$ was averaged over 100 different positions of the grid.

Ten on-lattice DLA clusters were grown in a circular geometry up to a size of 50000 particles. Initially the cluster points themselves were used as input to the box-counting algorithm. However, on examining the $(1 /(q-1)) \ln Z_{q}$ vs $\ln \epsilon$ plots, no extended scaling region was found (see Fig. 1). One can in fact see a systematic fall-off in the slope with decreasing $\epsilon$. Such behaviour has been observed previously [4]. A $D_{q}$ spectrum obtained from these plots would thus be suspect at best. We would therefore question the validity of the $D_{q}$ results quoted at the beginning of the letter.

The geometrical multifractality of the perimeter of the DLA clusters was next examined. As can be seen in Fig. 1, a scaling region of near constant slope exists in the $(1 /(q-1)) \ln Z_{q}$ vs $\ln \epsilon$ plots. Note that for $q<2$ a slight fall-off in the slope was still observed. A $D_{q}$ spectrum for $q \geqslant 0$ was subsequently obtained

\footnotetext{
${ }^{*}$ Corresponding author.
} 

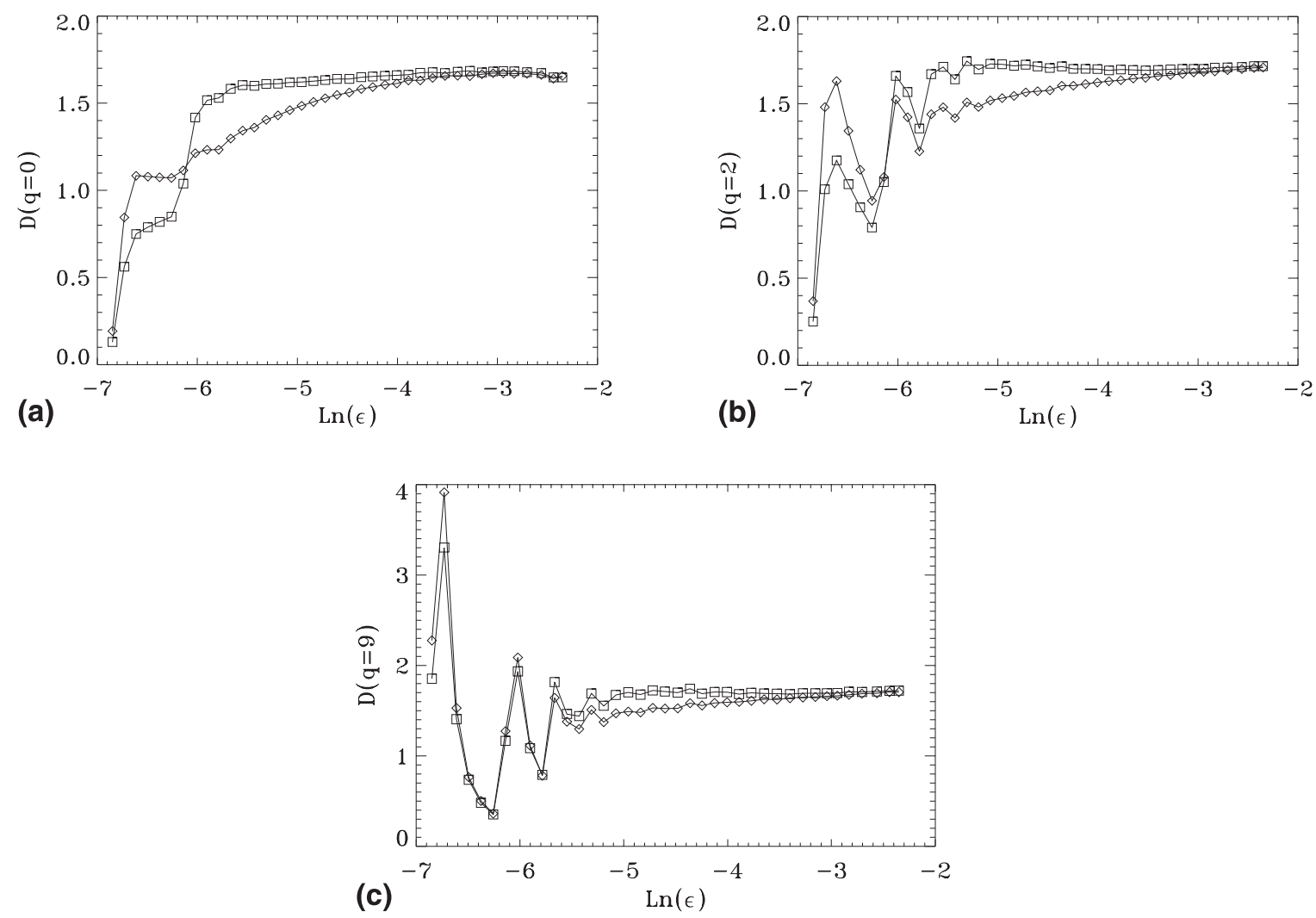

Fig. 1. Local slope of $(1 /(q-1)) \ln Z_{q}$ vs $\ln \epsilon$ plots using DLA cluster points (diamonds) and DLA perimeter points (squares) for: (a) $q=0$, (b) $q=2$ and (c) $q=9$.

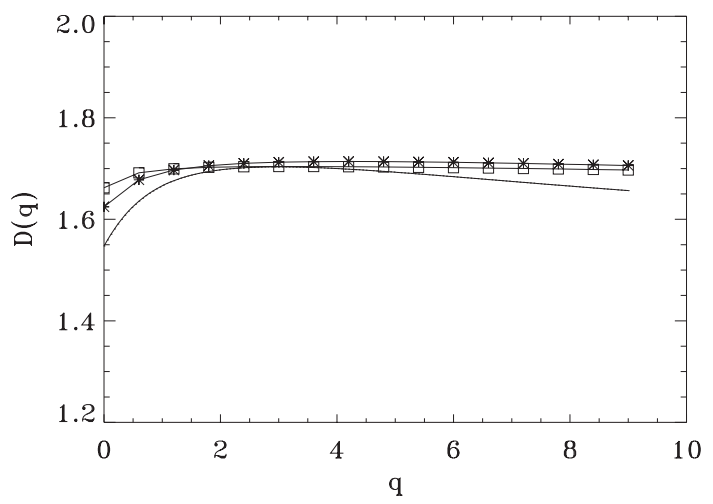

Fig. 2. $D_{q}$ spectra calculated using the perimeter sites of DLA clusters of sizes 50000 (squares), 5000 (asterisks) and 500 (line) particles.

from these plots and is shown in Fig. 2 (the $D_{q}$ spectra obtained from the perimeter points of 10 clusters of size 500 and 5000 particles is also shown). For approximately $q \geqslant 2$ the $D_{q}$ spectrum has converged and within numerical accuracy has a constant value of $1.70 \pm 0.01$. Significant finite-size effects are evident in our $D_{q}$ spectrum as $q \rightarrow 0$.

In summary, we have found that for the perimeter sites of DLA clusters, $Z_{q} \sim \epsilon^{(q-1) D_{q}}$ for approximately $q \geqslant 2$. For the cluster sizes considered, the cluster points themselves failed to satisfy this scaling law. The value obtained for $D_{2}$ is consistent with the value of $1.71 \pm 0.02$ for the dynamical dimension $D_{\mathrm{d}}$ [3]. For $q \geqslant 2$, the $D_{q}$ spectrum is also consistent with the previous results of Vicsek et al. [5] and Gang Li et al. [2]. 


\section{Acknowledgements}

We wish to acknowledge the financial support of Forbairt, the Irish National Funding Agency for Science and Technology.

\section{References}

[1] Argoul F, Arneodo A, Grasseau G, Swinney HL. Self-similarity of diffusion-limited aggregates and electrodeposition clusters. Phys Rev Lett 1988;61:2558-61.

[2] Li Gang, Sander LM, Meakin P. Comment on self-similarity of diffusion-limited aggregates and electrodeposition clusters. Phys Rev Lett 1989;63:1322.

[3] Meakin P. Diffusion-controlled cluster formation in 2-6 dimensional space. Phys Rev A 1983;27:1495-507.

[4] Argoul F, Arneodo A, Grasseau G, Swinney HL. Reply to comment on self-similarity of diffusion-limited aggregates and electrodeposition clusters. Phys Rev Lett 1989;63:1323.

[5] Vicsek T, Family F, Meakin P. Multifractal geometry of diffusion-limited aggregates. Europhys Lett 1990;12:217-22. 